\title{
PET/MRI Versus PET/CT for Whole-Body Staging: Results from a Single-Center Observational Study on 1,003 Sequential Examinations
}

\author{
Ole Martin*1, Benedikt M. Schaarschmidt*2, Julian Kirchner ${ }^{1}$, Saravanabavaan Suntharalingam² ${ }^{2}$ Johannes Grueneisen $^{2}$, \\ Aydin Demircioglu ${ }^{2}$, Philipp Heusch ${ }^{1}$, Harald H. Quick ${ }^{3,4}$, Michael Forsting ${ }^{2}$, Gerald Antoch ${ }^{1}$, Ken Herrmann ${ }^{5}$, \\ and Lale Umutlu ${ }^{2}$ \\ ${ }^{1}$ Department of Diagnostic and Interventional Radiology, University Dusseldorf, Medical Faculty, Dusseldorf, Germany; \\ ${ }^{2}$ Department of Diagnostic and Interventional Radiology and Neuroradiology, University Hospital Essen, University of \\ Duisburg-Essen, Essen, Germany; ${ }^{3}$ Erwin L. Hahn Institute for Magnetic Resonance Imaging, University of Duisburg-Essen, \\ Essen, Germany; ${ }^{4}$ High-Field and Hybrid MR Imaging, University Hospital Essen, University of Duisburg-Essen, Essen, \\ Germany; and ${ }^{5}$ Department of Nuclear Medicine, University Hospital Essen, University of Duisburg-Essen, Essen, \\ Germany
}

Our purpose was to investigate differences between PET/MRI and $\mathrm{PET} / \mathrm{CT}$ in lesion detection and classification in oncologic whole-body examinations and to investigate radiation exposure differences between the 2 modalities. Methods: In this observational single-center study, 1,003 oncologic examinations (918 patients; mean age, $57.8 \pm 14.4$ y) were included. Patients underwent PET/CT and subsequent PET/MRI $(149.8 \pm 49.7 \mathrm{~min}$ after tracer administration). Examinations were reviewed by radiologists and nuclear medicine physicians in consensus. Additional findings, characterization of indeterminate findings on $\mathrm{PET} / \mathrm{CT}$, and missed findings on PET/MRI, including their clinical relevance and effective dose of both modalities, were investigated. The McNemar test was used to compare lesion detection between the 2 hybrid imaging modalities $(P<0.001$, indicating statistical significance). Results: Additional information on PET/ MRI was reported for $26.3 \%(264 / 1,003)$ of examinations, compared with PET/CT $(P<0.001)$. Of these, additional malignant findings were detected in $5.3 \%(53 / 1,003)$, leading to a change in TNM staging in $2.9 \%(29 / 1,003)$ due to PET/MRI. Definite lesion classification of indeterminate PET/CT findings was possible in $11.1 \%(111 / 1,003)$ with PET/MRI. In $2.9 \%(29 / 1,003)$, lesions detected on PET/CT were not visible on PET/MRI. Malignant lesions were missed in $1.2 \%(12 / 1,003)$ on PET/MRI, leading to a change in TNM staging in $0.5 \%(5 / 1,003)$. The estimated mean effective dose for whole-body PET/CT amounted to $17.6 \pm 8.7 \mathrm{mSv}$, in comparison to $3.6 \pm 1.4 \mathrm{mSv}$ for PET/MRI, resulting in a potential dose reduction of $79.6 \%(P<0.001)$. Conclusion: PET/MRI facilitates staging comparable to that of PET/CT and improves lesion detectability in selected cancers, potentially helping to promote fast, efficient local and whole-body staging in 1 step, when additional MRI is recommended. Furthermore, younger patients may benefit from the reduced radiation exposure of PET/MRI.

Received Jul. 17, 2019; revision accepted Dec. 2, 2019.

For correspondence or reprints contact: Ole Martin, Department of Diagnostic and Interventional Radiology, University of Dusseldorf, Moorenstrasse 5, D-40225 Dusseldorf, Germany.

E-mail: ole.martin@med.uni-duesseldorf.de

${ }^{*}$ Contributed equally to this work.

Published online Dec. 5, 2019.

COPYRIGHT (c) 2020 by the Society of Nuclear Medicine and Molecular Imaging.
Key Words: simultaneous PET/MRI; oncologic imaging; lesion detection; PET/CT

J Nucl Med 2020; 61:1131-1136

DOI: 10.2967/jnumed.119.233940

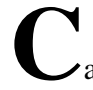

ancer remains one of the leading causes of death worldwide (1). Although morphologic imaging techniques to detect and monitor malignant diseases have advanced over the last few decades, limitations in their diagnostic accuracy remain. Functional imaging such as PET has improved the sensitivity and specificity in detecting malignant disease $(2,3)$. PET/CT combines high-resolution morphologic imaging with the sensitivity of PET, thus improving staging accuracy, optimizing therapy strategies, and improving patient outcome (4-7). Therefore, PET/CT has become a diagnostic cornerstone in various oncologic guidelines, most notably in lung cancer and lymphoma $(8,9)$. Two disadvantages of PET/CT comprise its low soft-tissue contrast and the additional radiation exposure to the radiopharmaceutical related to the CT component, necessary for morphologic correlation and attenuation correction. Both of these disadvantages may be overcome by exchanging the $\mathrm{CT}$ as the corresponding partner in hybrid imaging with MRI. Hence, the development of integrated PET/MRI scanners has been a long-term goal of researchers around the world. Despite technical challenges, 3 major benefits of this new modality have been postulated: MRI-related high soft-tissue contrast, the combination of functional and morphologic imaging, and the reduction of radiation exposure compared with PET/CT (10-13).

Initial studies investigating the potential advantages of PET/ MRI over PET/CT revealed promising results: PET/MRI leads to a markedly reduced radiation exposure $(14,15)$ and facilitates a high diagnostic performance for various oncologic diseases such as neuroendocrine tumors $(16,17)$, prostate cancer $(18)$, gynecologic tumors $(19,20)$, breast cancer $(21-23)$, and lymphoma $(14,20)$. However, in head and neck $(24,25)$ and lung cancer (26-28), an obvious advantage of PET/MRI has not been demonstrated. Still, these results have to be considered preliminary, as most studies are 
based on small patient cohorts (maximum, 100-150 patients). Furthermore, data pooling is difficult because of heterogeneous study endpoints (29). Because of the higher operational costs and the technical challenges of PET/MRI, this lack of high-quality data hinders a widespread introduction into clinical practice.

Therefore, the aim of this observational single-center study was to evaluate differences between PET/MRI and PET/CT for the detection and classification of lesions in different oncologic diseases.

\section{MATERIALS AND METHODS}

\section{Patients}

This observational study was funded by the German Research Foundation ("MR-PET for Medical Imaging," grant AN 397/3-1). The study followed institutional guidelines and was approved by the ethics committee of the Medical Faculty of the University of DuisburgEssen. Written informed consent was obtained from all patients before the examination.

The inclusion criteria comprised clinically indicated oncologic wholebody PET/CT and subsequent whole-body PET/MRI scans, both of which were from the skull base to the mid thighs. The exclusion criteria were contraindications to MRI (e.g., pacemakers) or to iodine or gadoliniumbased contrast medium.

\section{PET/CT}

All patients underwent an oncologic, clinically indicated wholebody PET/CT scan from skull base to mid thighs on a Biograph mCT (Siemens Healthcare). The choice of tracer depended on the clinical indication: ${ }^{18} \mathrm{~F}-\mathrm{FDG}$ in $71.7 \%(719 / 1,003$; mean activity, $258 \pm 50$ $\mathrm{MBq}),{ }^{68} \mathrm{Ga}$-PSMA in $13.2 \%$ (132/1,003; mean activity, $\left.112 \pm 26 \mathrm{MBq}\right)$, ${ }^{68} \mathrm{Ga}$-DOTATOC in 8.3\% (83/1,003; mean activity, $\left.66 \pm 16 \mathrm{MBq}\right),{ }^{124} \mathrm{I}-$ metaiodobenzylguanidine in $3.5 \%$ (35/1,003; mean activity, $47 \pm 9$ $\mathrm{MBq}),{ }^{124} \mathrm{I}$ in $1.6 \%(17 / 1,003$; mean activity, $32 \pm 8 \mathrm{MBq}),{ }^{18} \mathrm{~F}$-choline in $1.4 \%(14 / 1,003$; mean activity, $330 \pm 54 \mathrm{MBq})$, and ${ }^{18} \mathrm{~F}$-fluoride in $0.3 \%$ (3/1,003; mean activity, $154 \pm 4 \mathrm{MBq})$. In ${ }^{18} \mathrm{~F}-\mathrm{FDG}$ examinations, blood glucose levels were ensured to be lower than $150 \mathrm{mg} / \mathrm{dL}$. In cases of increased glucose levels, appropriate insulin medication was administered.

Low-dose $(n=187)$ and full-dose $(n=816)$ PET/CT scans were performed with automated tube voltage selection (CareKV preset of $120 \mathrm{kV}$; slice thickness, $5 \mathrm{~mm}$ ). Full-dose scans were performed with automated tube current modulation (CareDose 4D preset of $190 \mathrm{mAs}$ ) $70 \mathrm{~s}$ after the injection of a weight-dependent dose of contrast agent (Ultravist; Bayer Pharma). For low-dose scans, a CareDose 4D preset of $40 \mathrm{mAs}$ was chosen.

PET data were acquired at up to 7 bed positions ( 2 min per bed position). Attenuation-corrected PET images were reconstructed using the portal venous phase for full-dose scans and using low-dose CT data for low-dose scans. PET images were reconstructed using ordered-subset expectation maximization with 4 iterations and 8 subsets. A gaussian filter kernel with a full width at half maximum of $2 \mathrm{~mm}$ was used for postreconstruction filtering.

\section{PET/MRI}

After PET/CT, a subsequent whole-body PET/MRI scan was performed on a Biograph mMR (Siemens Healthcare) $149.8 \pm$ 49.7 min after tracer injection. The PET/MRI scans were obtained from skull base to mid thighs. All patients in this study received a macrocyclic gadolinium-based contrast agent for contrast-enhanced imaging (gadoterate meglumine, $0.05 \mathrm{mmol} / \mathrm{kg}$ of body weight [Guerbet], or gadobutrol, $0.1 \mathrm{mmol} / \mathrm{kg}$ of body weight [Bayer Pharma]). At each bed position, 4 MRI sequences were performed. The first, for MRIbased attenuation correction, was a coronal 3-dimensional Dixon volumeinterpolated breath-hold examination. The second was a transverse
T1-weighted sequence. Until 2015, this was a T1-weighted fast lowangle shot sequence after contrast administration with fat saturation. After a software update, a transverse T1-weighted volume-interpolated breath-hold examination was used because of faster acquisition times and higher soft-tissue contrast. The third sequence was a transverse T2weighted half-Fourier acquired single-shot turbo spin-echo sequence. The fourth, for diffusion-weighted imaging, was a transverse thoracic echo planar imaging sequence with 3 b-values $(0,500,1,000)$ during free breathing.

In whole-body imaging, only contrast-enhanced T1-weighted sequences were obtained. Depending on the clinical indication, additional high-resolution MRI sequences were acquired for evaluation of local tumor extent (e.g., in prostate or breast cancer) or for metastasis detection (e.g., for the liver in gastrointestinal tumors), when needed. In the latter instance, for example, additional dynamic sequences of the liver were added.

PET data were acquired in list mode without respiratory gating (up to 5 bed positions, 4 min per bed position). Three-dimensional iterative image reconstruction was performed ( 3 iterations, 21 subsets, gaussian filter of $4 \mathrm{~mm}$ ).

\section{Image Analysis and Data Evaluation}

All PET/CT and PET/MRI examinations were evaluated by boardcertified nuclear medicine physicians and board-certified radiologists. In the PET/MRI report, the following findings were noted and analyzed: additional findings on PET/MRI that were missed on PET/CT and their most probable diagnosis, additional but indeterminate findings on PET/ MRI requiring additional examinations or follow-up, classification of indeterminate findings on PET/CT by PET/MRI, and missed findings on PET/MRI in comparison to PET/CT.

To evaluate the nature of these lesions (benign vs. malignant), clinical and histologic reports, characteristic imaging findings on the subsequent PET/MRI examination, and additional radiologic examinations, including follow-up examinations, were used as a reference standard. According to the reference standard, changes in tumor stage on PET/MRI were analyzed. Based on radiotracer activity, the effective dose (ED) of the PET part of the examination was calculated using the whole-body ED coefficient recommended by Andersson et al. (30). Using DICOMstructured reports, ED due to the CT part of the PET/CT examination was estimated according to a method described by Christner et al. (31).

\section{Statistics}

An explorative data analysis was performed for patient and examination characteristics. All additional findings on PET/MRI, findings that were considered indeterminate on PET/CT and requiring follow-up but capable of being further classified on PET/MRI, and findings missed on PET/MRI were analyzed. Changes in TNM staging were evaluated. Differences in lesion detection were compared using the McNemar test. Estimated mean EDs were compared using the paired $t$ test. A $P$ value of less than 0.001 indicated statistical significance. Statistical analysis was performed using SPSS Statistics, version 24 (IBM Corp.).

\section{RESULTS}

\section{Patient Characteristics}

Between March 2012 and June 2018, 4,949 PET/MRI examinations were performed at our institution on 4,659 patients. Of these examinations, 3,946 were excluded for various reasons (Fig. 1). Therefore, 1,003 PET/MRI examinations on 918 patients (mean age, $57.8 \pm 14.4$ y; 400 female, 518 male) were eligible for analysis, resulting in 846 examinations for tumor staging and 157 for response assessment. The oncologic indications were lung cancer $(17.9 \%, 180 / 1,003)$, gastrointestinal cancer or neuroendocrine tumors $(17.2 \%, 173 / 1,003)$, gynecologic or breast cancer 


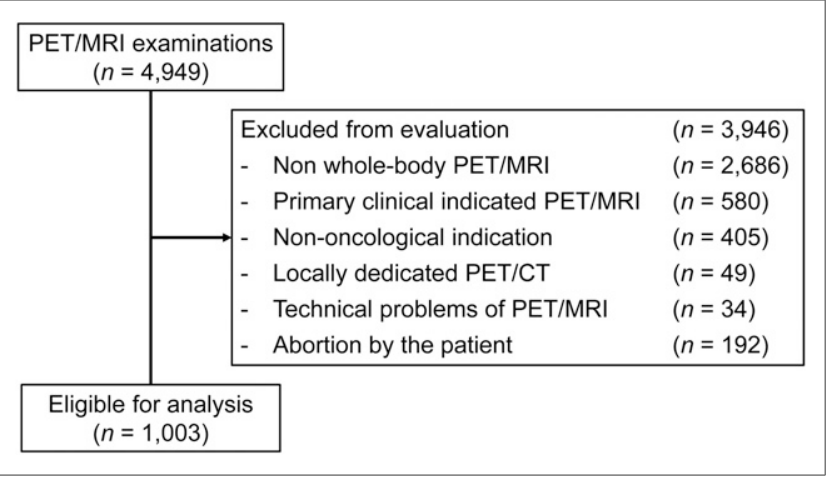

FIGURE 1. CONSORT diagram. From 4,949 examinations overall, 3,946 cases were excluded from analyses, resulting in 1,003 evaluable examinations.

$(14.8 \%, 148 / 1,003)$, prostate cancer $(14.8 \%, 148 / 1,003)$, lymphoma $(12.0 \%, 120 / 1,003)$, melanoma $(8.7 \%, 87 / 1,003)$, head and neck cancer $(8.0 \%, 80 / 1,003)$, cancer of unknown primary $(4.3 \%, 43 / 1,003)$, and malignant bone disease $(2.4 \%, 24 / 1,003)$.

\section{Differences in Lesion Detection Between PET/MRI and PET/CT}

According to the McNemar test, significantly more lesions were detected on PET/MRI than on PET/CT $(P<0.001)$. In $15.4 \%$ $(155 / 1.003)$ of all examinations, additional lesions were identified on PET/MRI that were not detected on PET/CT (Fig. 2). Followup imaging was available for $66.5 \%$ (103/155). In an additional $11.0 \%(17 / 155)$ of examinations, equivocal PET/CT lesions could be clearly classified on PET/MRI without the need for further investigation, providing a reference standard for 120 of 155 lesions. Among them, PET/MRI enabled a definite classification for 88 of 120 lesions, comprising a correct classification for $66.7 \%$ (80/120; 53 malignant and 27 benign lesions) and an incorrect classification for $6.7 \%(8 / 120 ; 7$ malignant and 1 benign). For $26.7 \%(32 / 120)$, the additionally detected lesions were considered indeterminate on PET/MRI, requiring further investigation. According to the reference standard, $28.1 \%(9 / 32)$ of all additionally detected but indeterminate lesions on PET/MRI were shown to be malignant and $71.9 \%(23 / 32)$ to be benign.

Overall, 24.2\% (29/120) of the lesions newly detected on PET/ MRI led to a correction in the TNM stage (T0 to T + in 9 cases; $\mathrm{N} 0$ to $\mathrm{N}+$ in 3 cases; $\mathrm{M} 0$ to $\mathrm{M}+$ in 17 cases). In 2 of $120(1.7 \%)$ patients, an incorrect lesion classification on PET/MRI led to an incorrect upstaging from $\mathrm{T} 0$ to $\mathrm{T}+$ for a lung cancer patient and from $\mathrm{N} 0$ to $\mathrm{N}+$ for a breast cancer patient.

The additional findings were observed mostly in patients with malignant bone disease $(33.3 \%, 8 / 24)$, lung cancer $(14.4 \%, 26 / 180)$, prostate cancer $(12.2 \%, 18 / 148)$, gynecologic or breast cancer $(12.2 \%$, $18 / 148)$, gastrointestinal cancer or neuroendocrine tumors $(11.0 \%$, $19 / 173)$, or malignant melanoma $(10.3 \%, 9 / 87)$. Additional malignant lesions on PET/MRI were detected mostly in patients with malignant bone disease $(12.5 \%, 3 / 24)$, lung cancer $(7.8 \%, 14 / 180)$, prostate cancer $(7.4 \%, 11 / 148)$ (Fig. 3), and gastrointestinal cancer or neuroendocrine tumors $(6.4 \%, 11 / 173)$.

Additional findings on PET/MRI led to a TNM stage correction for $5.4 \%$ of prostate cancer cases (8/148; upstaging from $\mathrm{T} 0$ to $\mathrm{T}+$ in 4 cases and $\mathrm{M} 0$ to $\mathrm{M}+$ in 4 cases), $4.6 \%$ of malignant melanoma cases (4/87; upstaging from N0 to $\mathrm{N}+$ in 1 case and M0 to $\mathrm{M}+$ in 3 cases), and $3.5 \%$ of gastrointestinal cancer or neuroendocrine tumor cases (6/ 173; upstaging from $\mathrm{T} 0$ to $\mathrm{T}+$ in 2 cases and $\mathrm{M} 0$ to $\mathrm{M}+$ in 4 cases).

In $2.9 \%(29 / 1,003)$ of the examinations, PET/MRI did not detect lesions that were found on PET/CT. According to the reference standard, which was available for $86.2 \%(25 / 29)$ of the examinations, lesions missed on PET/MRI were considered malignant in $1.2 \%(12 / 1,003)$ of the cases and as benign in $1.3 \%$ $(13 / 1,003)$. The missed malignant lesions comprised small lung nodules ( $n=8$, size $<10 \mathrm{~mm}$; Fig. 4$)$, lymph node metastases $(n=$ 2 ), a bone metastasis $(n=1)$, and malignant tissue of the hypopharynx $(n=1)$, leading to an overall change in TNM stage in 5 cases $(0.5 \%)$ due to an increase in $\mathrm{M}$ stage (M0 to $\mathrm{M}+$ ). In all other cases, the malignant lesions not detected by PET/MRI did not lead to changes in TNM stage because diffuse metastatic spread was present. The missed benign findings comprised lung granuloma $(n=8)$ and unspecific tracer uptake in bone $(n=3)$, liver $(n=1)$, and pharynx $(n=1)$.

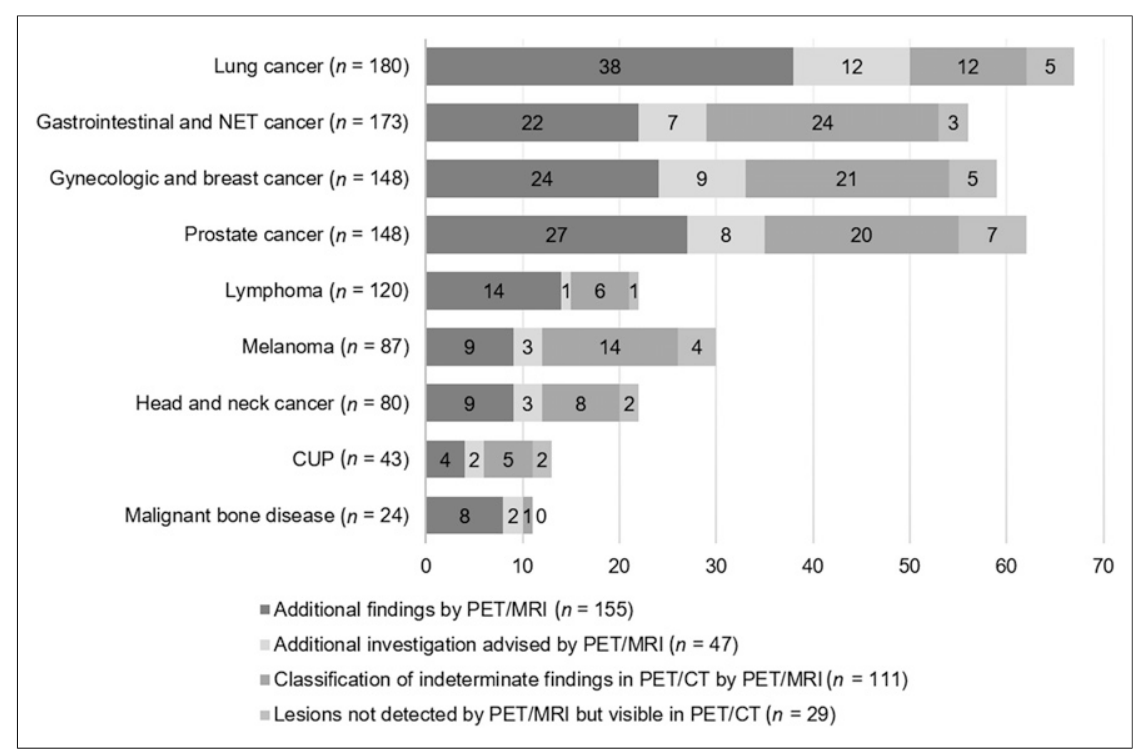

FIGURE 2. Additional information from PET/MRI for different cancer entities. CUP = cancer of unknown primary; NET = neuroendocrine tumor.

\section{Classification of Indeterminate Findings on PET/CT by PET/MRI}

In total, 111 findings in 1,003 examinations $(11.1 \%)$ were classified as indeterminate on PET/CT. Follow-up imaging was available for $62.2 \%(69 / 111)$, and in an additional $23.4 \%(26 / 111)$, the lesion could be clearly classified on PET/MRI without the need for further investigation, providing a reference standard for 95 lesions. PET/MRI correctly classified the findings that were indeterminate on PET/CT in $98.9 \%$ of the cases $(94 / 95 ; 29$ malignant, 65 benign; Fig. 5). In 1 case $(1.1 \%)$, an indeterminate lesion on PET/CT was classified incorrectly on PET/MRI: a suggestive liver lesion in a breast cancer patient that was incorrectly classified as a benign hemangioma turned out to be malignant on follow-up PET/CT after $753 \mathrm{~d}$. This finding led to incorrect TNM staging (M0 instead of further investigation or follow-up). 


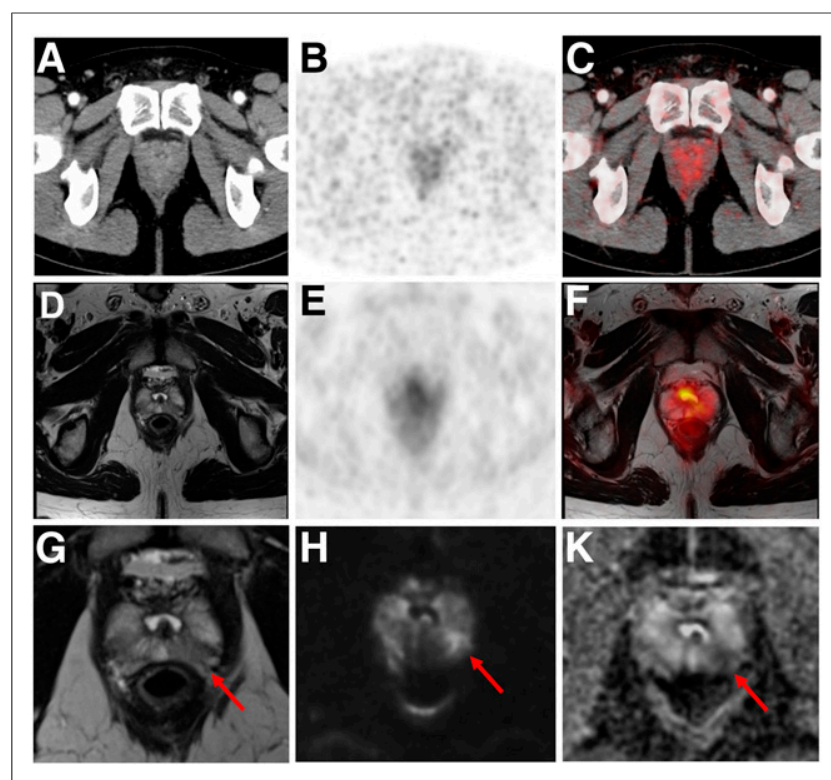

FIGURE 3. Additional finding from PET/MRI for 51-y-old man with prostate cancer. Contrast-enhanced CT (A), PET (B), and fused ${ }^{68} \mathrm{Ga}-$ PSMA PET/CT (C) images are displayed in comparison to T2-weighted MRI (D and G), PET (E), and fused ${ }^{68} \mathrm{Ga}-\mathrm{PSMA}$ PET/MR (F) images, as well as diffusion-weighted images (b1000 $[\mathrm{H}]$ and $A D C$ map $[K])$. Suggestive lesion (arrows) in left peripheral zone was missed by CT and PET on PET/CT and by PET on PET/MRI because of its small size in homogeneous soft tissue. However, restricted diffusion of soft-tissue mass is indicative of malignancy. Additional biopsy confirmed diagnosis afterward

Otherwise, PET/MRI led to a correction of the TNM stage according to the reference standard in $7.4 \%$ of the cases $(7 / 95$, upstaging from $\mathrm{T} 0$ to $\mathrm{T}+$ in 1 case and $\mathrm{M} 0$ to $\mathrm{M}+$ in 2 cases; downstaging from $\mathrm{T}+$ to $\mathrm{T} 0$ in 2 cases and $\mathrm{M}+$ to $\mathrm{M} 0$ in 2 cases).

\section{Radiation Exposure}

The mean ED of all whole-body PET/CT examinations amounted to $17.6 \pm 8.7 \mathrm{mSv}$, with PET accounting for $3.6 \pm 1.4 \mathrm{mSv}(20.5 \%)$. Because of the lack of additional radiation exposure for attenuation correction and morphologic imaging in PET/MRI, the radiation exposure could be significantly reduced by $83.2 \%(3.5 \pm 1.4 \mathrm{mSv})$

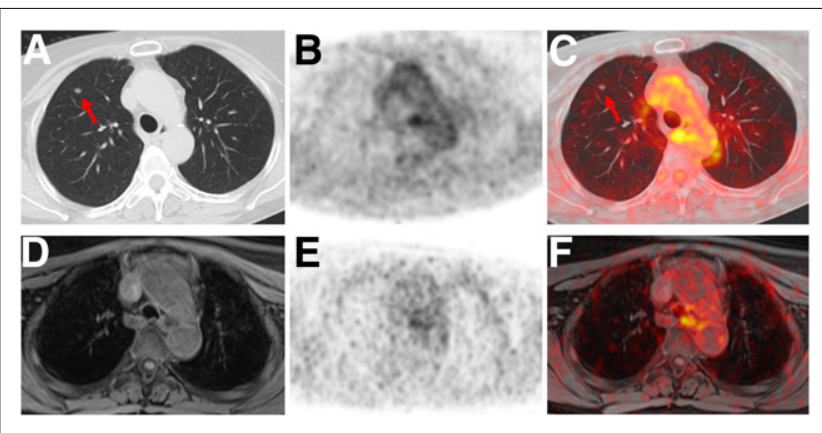

FIGURE 4. Finding missed on PET/MRI for 77-y-old woman with ovarian carcinoma. Contrast-enhanced CT (A), PET (B), and fused ${ }^{18} \mathrm{~F}-\mathrm{FDG}$ PET/CT (C) images are displayed in comparison with T1-weighted MRI (D), PET (E), and fused ${ }^{18}$ F-FDG PET/MRI (F) images. Small lung metastasis (arrows) in right upper lobe that is missed by MRI, as well as by PET on PET/MRI and PET/CT, because of its small size is clearly visible on CT. Follow-up CT confirmed malignancy after $78 \mathrm{~d}$.
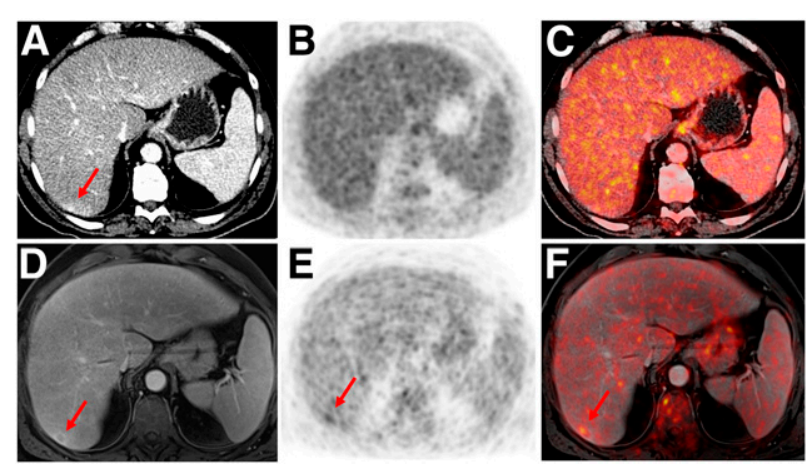

FIGURE 5. Indeterminate lesion on PET/CT classified by PET/MRI for 53-y-old man with lung cancer. Contrast-enhanced CT (A), PET (B), and fused ${ }^{18} \mathrm{~F}-\mathrm{FDG}$ PET/CT (C) images are displayed in comparison with contrast-enhanced T1-weighted MRI (D), PET, and fused ${ }^{18} \mathrm{~F}-\mathrm{FDG}$ PET/MRI (F) images. In CT (A), hyperdense, subcentimeter liver lesion (arrows) in segment VII is suggestive of transient hepatic attenuation difference or small hemangioma. As malignancy cannot be excluded, it needs further investigation. On PET/MRI, lesion is clearly classified as metastasis because of contrast enhancement and tracer uptake due to later acquisition time point. Follow-up CT confirmed diagnosis after $78 \mathrm{~d}$.

through the use of PET/MRI rather than full-dose PET/CT (20.8 \pm $7.0 \mathrm{mSv})$ and by $36.1 \%(3.9 \pm 1.3 \mathrm{mSv})$ through the use of PET/MRI rather than low-dose PET/CT $(6.1 \pm 1.6 \mathrm{mSv})$ (both $P<0.001)$.

\section{DISCUSSION}

The results of this first single-center observational study on over 1,000 PET/CT and PET/MRI examinations carry 3 important messages. The first is that PET/MRI improves lesion detection for selected cancers and potentially reduces the need for additional examinations in comparison to PET/CT. The second is that the number of missed malignant lesions, particularly lung metastases, on PET/MRI is negligibly small $(0.8 \%)$, contradicting previous beliefs in the need for additional CT imaging of the chest. The third is that our results confirm and underline the potential for a clinically relevant reduction of radiation exposure by using PET/ MRI instead of PET/CT - a finding that is of particular interest for younger patients, considering the cumulative radiation dose for staging, therapy monitoring, and aftercare.

Integrated PET/MRI systems have been successfully introduced into scientific and clinical applications over the past $8 \mathrm{y}$, leveraging hybrid imaging onto a new platform of simultaneous acquisition of complementary metabolic, functional, and morphologic information based on simultaneously acquired PET and MR datasets. Most early clinical PET/MRI studies focused on comparing its diagnostic performance with that of PET/CT or conventional imaging (MRI) and found potential benefits in tumor staging and therapy management for various tumor entities $(11,12,27,32-36)$. The exceptional soft-tissue contrast and the inherent multifunctionality of MRI, in terms of the potential for multiparametric MRI, were shown to facilitate superior lesion detection and improve diagnostic accuracy (18,37-39). However, these promising preliminary findings were based on small patient cohorts. In particular, a preliminary study by Catalano et al. detected changes in clinical management in up to $18 \%$ of patients, although these results were based on a highly selected patient cohort using ${ }^{18} \mathrm{~F}-\mathrm{FDG}$ examinations alone, as well as a considerable number of non-contrast-enhanced PET/CT examinations (13). Our results support and validate these results in revealing 
a higher rate for detecting additional malignant lesions on PET/MRI, mainly for prostate cancer, head and neck cancer, gynecologic or breast cancer, and gastrointestinal cancer or neuroendocrine tumors, most likely due to the enhanced soft-tissue contrast of MRI. In these tumor entities, primary lesions and recurrences can be detected more reliably on PET/MRI than on PET/CT. Additionally, multiparametric MRI protocols lead to a significantly better evaluation of suggestive abdominal lesions in these patients (40). In prostate cancer imaging, the advantages of local tumor staging by MRI and distant-metastasis detection by whole-body ${ }^{68} \mathrm{Ga}$-PSMA PET/CT could be combined into a 1-stop-shop PET/MRI examination, leading to enhanced patient comfort and a considerable acceleration of the diagnostic workup $(41,42)$. Apart from detecting additional lesions, PET/MRI also improves classification of lesions rated as indeterminate on PET/CT, hence potentially reducing the need for additional imaging procedures. As demonstrated in previous investigations $(32,43,44)$, this improvement was particularly true for small lesions in liver, kidney, and prostate because of the higher soft-tissue contrast of T2-weighted and contrast-enhanced T1-weighted imaging, as well as the additional diagnostic value of diffusion-weighted imaging. Thus, because of the higher diagnostic certainty in selected cancers and lesions, PET/MRI bears the potential to require fewer examinations than PET/CT.

One major concern that has limited the use of PET/MRI as an alternative to PET/CT for whole-body staging has been the inferiority of MRI in lung nodule detection $(45,46)$. Previous investigations on smaller patient cohorts ( $\leq 121$ patients) showed comparable diagnostic competence between PET/MRI and PET/CT for lung lesions larger than $10 \mathrm{~mm}$ and inferiority for PET/MRI for lesions smaller than $10 \mathrm{~mm}$ (45). Nevertheless, further investigations by Raad et al. and Sawicki et al. showed that most (97\%) lung nodules missed on PET/MRI either resolved or remained stable on follow-up, indicative of their benignity and leaving the potential effects on therapeutic management changes questionable $(46,47)$. Amplifying prior study cohorts by 8 - to 10 -fold to 1,003 PET/MRI examinations, our study found that the number of missed malignant lung nodules totalled 8 ( $0.8 \%$ of all examinations), hence underlining the high diagnostic potential of PET/MRI as an alternative to PET/CT, considering the negligibly low number of undetected lung metastases. Furthermore, new MRI sequences, such as ultrashortecho-time sequences, are expected to improve lung nodule detection in comparison to established lung MRI protocols, thus improving the sensitivity of PET/MRI even further (48). Another demur that had a negative impact on the clinical implementation of PET/MRI was the misconception that excessively long examination times rendered PET/MRI an ineffective research tool incompatible for clinical use. However, this misconception has been proven wrong in many studies demonstrating the comparability of PET/MRI and PET/CT for whole-body staging when specific fast protocols are used (49-52), although further research is needed to elucidate the significance of whole-body diffusion-weighted imaging, as well as contrast agent administration in whole-body PET/MRI protocols.

One major benefit of PET/MRI for patient care is the significant reduction of radiation exposure. Similar to previous publications on smaller cohorts, our results confirm the potential for a mean dose reduction of $83.2 \%$ when compared with full-dose PET/CT imaging. Because possible genotoxic effects of MRI have been discussed by various researchers, the quality of most studies has been a serious matter of concern (53). Furthermore, a negative study by Fatahi et al. in 2016 on individuals repeatedly exposed to examinations on 7-T MRI scanners seemed to indicate that the clinical relevance of genotoxic effects by MRI is negligible (54). Hence, in view of the high diagnostic accuracy of PET/MRI, it may be particularly beneficial in pediatric patients and adolescents, considering the cumulative radiation dose for multiple examinations for staging, therapy monitoring, and aftercare (9). Acknowledging the advantages of PET/MRI for pediatric patients, the German Society of Pediatric Oncology and Haematology recently introduced PET/MRI as an alternative to PET/CT for imaging patients with Hodgkin lymphoma (55).

Therefore, PET/MRI might be considered an important supplement to PET/CT. Although PET/CT will still be considered the oncologic workhorse, patients might profit from the diagnostic advantage due to the improved soft-tissue contrast of MRI, as well as from the improved diagnostic workflow for certain cancers. Furthermore, pediatric patients and young adults may profit from the markedly reduced radiation exposure, especially in oncologic diseases requiring repeated PET imaging, such as lymphoma.

This study had some limitations. To reduce radiation exposure, PET/CT and PET/MRI were performed sequentially after a single radiotracer injection. Therefore, PET/MRI examinations had a later acquisition timepoint than PET/CT, resulting in an increased sensitivity for metastases at the expense of specificity, such as in small lymph nodes (56). Furthermore, PET/MRI protocols evolved during the course of the study as new software updates by the vendor provided more advanced MRI sequences, which were implemented to guarantee the best possible image quality.

\section{CONCLUSION}

Our results demonstrate the comparability and potential benefit of PET/MRI toward PET/CT, with an improved detection rate in selected cancers and an overall reduced radiation exposure, which may be particularly beneficial in pediatric and adolescent patients. Future studies addressing the technical and operational challenges of PET/MRI, as well as involving different vendors and even larger cohorts, will hopefully further pave the way toward a widespread introduction of PET/MRI into clinical patient care.

\section{DISCLOSURE}

No potential conflict of interest relevant to this article was reported.

\section{KEY POINTS}

QUESTION: Are there differences between PET/MRI and PET/CT in lesion detection and classification in oncologic imaging?

PERTINENT FINDINGS: In this 6-y observational study with over 1,000 investigated examinations, PET/MRI improved lesion detection and classification in comparison to PET/CT in oncology, thus changing TNM staging. Furthermore, PET/MRI significantly reduced ionizing radiation in comparison to $\mathrm{PET} / \mathrm{CT}$.

IMPLICATIONS FOR PATIENT CARE: PET/MRI reduces the need for additional examinations for tumor staging. The radiation exposure reduction may be highly beneficial, particularly for pediatric and adolescent patients.

\section{REFERENCES}

1. Torre LA, Bray F, Siegel RL, Ferlay J, Lortet-Tieulent J, Jemal A. Global cancer statistics, 2012. CA Cancer J Clin. 2015;65:87-108.

2. Lardinois D, Weder W, Hany TF, et al. Staging of non-small-cell lung cancer with integrated positron-emission tomography and computed tomography. $N$ Engl J Med. 2003;348:2500-2507. 
3. Fischer B, Lassen U, Mortensen J, et al. Preoperative staging of lung cancer with combined PET-CT. N Engl J Med. 2009;361:32-39.

4. Beyer T, Townsend DW, Brun T, et al. A combined PET/CT scanner for clinical oncology. J Nucl Med. 2000;41:1369-1379.

5. Antoch G, Vogt FM, Freudenberg LS, et al. Whole-body dual-modality PET/CT and whole-body MRI for tumor staging in oncology. JAMA. 2003;290:3199-3206.

6. Radford J, Illidge T, Counsell N, et al. Results of a trial of PET-directed therapy for early-stage Hodgkin's lymphoma. N Engl J Med. 2015;372:1598-1607.

7. Mehanna H, Wong WL, McConkey CC, et al. PET-CT surveillance versus neck dissection in advanced head and neck cancer. N Engl J Med. 2016;374:1444-1454.

8. Chao F, Zhang H. PET/CT in the staging of the non-small-cell lung cancer. $J$ Biomed Biotechnol. 2012;2012:783739.

9. Cheson BD, Fisher RI, Barrington SF, et al. Recommendations for initial evaluation, staging, and response assessment of Hodgkin and non-Hodgkin lymphoma: the Lugano classification. J Clin Oncol. 2014;32:3059-3068.

10. Quick HH. Integrated PET/MR. J Magn Reson Imaging. 2014;39:243-258.

11. Buchbender C, Heusner TA, Lauenstein TC, Bockisch A, Antoch G. Oncologic PET/MRI, part 1: tumors of the brain, head and neck, chest, abdomen, and pelvis. J Nucl Med. 2012;53:928-938.

12. Buchbender C, Heusner TA, Lauenstein TC, Bockisch A, Antoch G. Oncologic PET/MRI, part 2: bone tumors, soft-tissue tumors, melanoma, and lymphoma. $J$ Nucl Med. 2012;53:1244-1252.

13. Catalano OA, Rosen BR, Sahani DV, et al. Clinical impact of PET/MR imaging in patients with cancer undergoing same-day PET/CT: initial experience in 134 patients-a hypothesis-generating exploratory study. Radiology. 2013;269:857-869.

14. Grueneisen J, Sawicki LM, Schaarschmidt BM, et al. Evaluation of a fast protocol for staging lymphoma patients with integrated PET/MRI. PLoS One. 2016;11:e0157880.

15. Schafer JF, Gatidis S, Schmidt H, et al. Simultaneous whole-body PET/MR imaging in comparison to PET/CT in pediatric oncology: initial results. Radiology. 2014;273:220-231.

16. Blanchet EM, Millo C, Martucci V, Maass-Moreno R, Bluemke DA, Pacak K. Integrated whole-body PET/MRI with ${ }^{18} \mathrm{~F}-\mathrm{FDG},{ }^{18} \mathrm{~F}-\mathrm{FDOPA}$, and ${ }^{18} \mathrm{~F}$-FDA in paragangliomas in comparison with PET/CT: NIH first clinical experience with a singleinjection, dual-modality imaging protocol. Clin Nucl Med. 2014;39:243-250.

17. Hope TA, Pampaloni MH, Nakakura E, et al. Simultaneous ${ }^{68}$ Ga-DOTA-TOC PET/MRI with gadoxetate disodium in patients with neuroendocrine tumor. Abdom Imaging. 2015;40:1432-1440.

18. Wetter A, Lipponer C, Nensa F, et al. Evaluation of the PET component of simultaneous $\left[{ }^{18} \mathrm{~F}\right]$ choline PET/MRI in prostate cancer: comparison with $\left[{ }^{18} \mathrm{~F}\right]$ choline PET/CT. Eur J Nucl Med Mol Imaging. 2014;41:79-88.

19. Bagade S, Fowler KJ, Schwarz JK, Grigsby PW, Dehdashti F. PET/MRI evaluation of gynecologic malignancies and prostate cancer. Semin Nucl Med. 2015;45:293-303.

20. Rosenkrantz AB, Friedman K, Chandarana H, et al. Current status of hybrid PET/MRI in oncologic imaging. AJR. 2016;206:162-172.

21. Taneja S, Jena A, Goel R, Sarin R, Kaul S. Simultaneous whole-body ${ }^{18}$ F-FDG PET-MRI in primary staging of breast cancer: a pilot study. Eur J Radiol. 2014; 83:2231-2239.

22. Pace L, Nicolai E, Luongo A, et al. Comparison of whole-body PET/CT and PET/ MRI in breast cancer patients: lesion detection and quantitation of ${ }^{18} \mathrm{~F}$-deoxyglucose uptake in lesions and in normal organ tissues. Eur J Radiol. 2014;83:289-296.

23. Sawicki LM, Grueneisen J, Schaarschmidt BM, et al. Evaluation of ${ }^{18}$ F-FDG PET/MRI, ${ }^{18}$ F-FDG PET/CT, MRI, and CT in whole-body staging of recurrent breast cancer. Eur J Radiol. 2016;85:459-465.

24. Queiroz MA, Huellner MW. PET/MR in cancers of the head and neck. Semin Nucl Med. 2015;45:248-265.

25. Schaarschmidt BM, Heusch $\mathrm{P}$, Buchbender C, et al. Locoregional tumour evaluation of squamous cell carcinoma in the head and neck area: a comparison between MRI, PET/CT and integrated PET/MRI. Eur J Nucl Med Mol Imaging. 2016;43:92-102.

26. Flechsig P, Mehndiratta A, Haberkorn U, Kratochwil C, Giesel FL. PET/MRI and PET/CT in lung lesions and thoracic malignancies. Semin Nucl Med. 2015;45:268-281.

27. Heusch P, Buchbender C, Kohler J, et al. Thoracic staging in lung cancer: prospective comparison of ${ }^{18} \mathrm{~F}-\mathrm{FDG}$ PET/MR imaging and ${ }^{18} \mathrm{~F}-\mathrm{FDG}$ PET/CT. $J$ Nucl Med. 2014;55:373-378.

28. Schaarschmidt BM, Grueneisen J, Metzenmacher M, et al. Thoracic staging with ${ }^{18}$ F-FDG PET/MR in non-small cell lung cancer: does it change therapeutic decisions in comparison to ${ }^{18} \mathrm{~F}$-FDG PET/CT? Eur Radiol. 2017;27:681-688.

29. Spick C, Herrmann K, Czernin J. ${ }^{18}$ F-FDG PET/CT and PET/MRI perform equally well in cancer: evidence from studies on more than 2,300 patients. $J$ Nucl Med. 2016;57:420-430.

30. Andersson M, Johansson L, Minarik D, Leide-Svegborn S, Mattsson S. Effective dose to adult patients from 338 radiopharmaceuticals estimated using ICRP biokinetic data, ICRP/ICRU computational reference phantoms and ICRP 2007 tissue weighting factors. EJNMMI Phys. 2014;1:9.

31. Christner JA, Kofler JM, McCollough CH. Estimating effective dose for CT using dose-length product compared with using organ doses: consequences of adopting International Commission on Radiological Protection publication 103 or dual-energy scanning. AJR. 2010;194:881-889.

32. Beiderwellen K, Gomez B, Buchbender C, et al. Depiction and characterization of liver lesions in whole body $\left[{ }^{18}\right.$ F]-FDG PET/MRI. Eur J Radiol. 2013;82: e669-e675.

33. Erfanian Y, Grueneisen J, Kirchner J, et al. Integrated ${ }^{18} \mathrm{~F}-\mathrm{FDG}$ PET/MRI compared to MRI alone for identification of local recurrences of soft tissue sarcomas: a comparison trial. Eur J Nucl Med Mol Imaging. 2017;44:1823-1831.

34. Grueneisen J, Nagarajah J, Buchbender C, et al. Positron emission tomography/ magnetic resonance imaging for local tumor staging in patients with primary breast cancer: a comparison with positron emission tomography/computed tomography and magnetic resonance imaging. Invest Radiol. 2015;50:505-513.

35. Grueneisen J, Schaarschmidt BM, Beiderwellen K, et al. Diagnostic value of diffusion-weighted imaging in simultaneous ${ }^{18} \mathrm{~F}-\mathrm{FDG}$ PET/MR imaging for wholebody staging of women with pelvic malignancies. J Nucl Med. 2014;55:1930-1935.

36. Sekine T, Barbosa FG, Delso G, et al. Local resectability assessment of head and neck cancer: positron emission tomography/MRI versus positron emission tomography/CT. Head Neck. 2017;39:1550-1558.

37. Herrmann K, Queiroz M, Huellner MW, et al. Diagnostic performance of FDGPET/MRI and WB-DW-MRI in the evaluation of lymphoma: a prospective comparison to standard FDG-PET/CT. BMC Cancer. 2015;15:1002.

38. Sawicki LM, Deuschl C, Beiderwellen K, et al. Evaluation of ${ }^{68} \mathrm{Ga}$-DOTATOC $\mathrm{PET} / \mathrm{MRI}$ for whole-body staging of neuroendocrine tumours in comparison with ${ }^{68}$ Ga-DOTATOC PET/CT. Eur Radiol. 2017;27:4091-4099.

39. Hartenbach M, Hartenbach S, Bechtloff W, et al. Combined PET/MRI improves diagnostic accuracy in patients with prostate cancer: a prospective diagnostic trial. Clin Cancer Res. 2014;20:3244-3253.

40. Luna A, Pahwa S, Bonini C, Alcala-Mata L, Wright KL, Gulani V. Multiparametric MR imaging in abdominal malignancies. Magn Reson Imaging Clin N Am. 2016;24:157-186.

41. Siddiqui MM, Rais-Bahrami S, Turkbey B, et al. Comparison of MR/ultrasound fusion-guided biopsy with ultrasound-guided biopsy for the diagnosis of prostate cancer. JAMA. 2015;313:390-397.

42. Shen G, Deng H, Hu S, Jia Z. Comparison of choline-PET/CT, MRI, SPECT, and bone scintigraphy in the diagnosis of bone metastases in patients with prostate cancer: a meta-analysis. Skeletal Radiol. 2014;43:1503-1513.

43. Schaarschmidt BM, Grueneisen J, Heusch P, et al. Does ${ }^{18}$ F-FDG PET/MRI reduce the number of indeterminate abdominal incidentalomas compared with ${ }^{18}$ F-FDG PET/CT? Nucl Med Commun. 2015;36:588-595.

44. Niekel MC, Bipat S, Stoker J. Diagnostic imaging of colorectal liver metastases with CT, MR imaging, FDG PET, and/or FDG PET/CT: a meta-analysis of prospective studies including patients who have not previously undergone treatment. Radiology. 2010;257:674-684.

45. Sawicki LM, Grueneisen J, Buchbender C, et al. Comparative performance of ${ }^{18} \mathrm{~F}$-FDG PET/MRI and ${ }^{18} \mathrm{~F}$-FDG PET/CT in detection and characterization of pulmonary lesions in 121 oncologic patients. J Nucl Med. 2016;57:582-586.

46. Raad RA, Friedman KP, Heacock L, Ponzo F, Melsaether A, Chandarana H. Outcome of small lung nodules missed on hybrid PET/MRI in patients with primary malignancy. J Magn Reson Imaging. 2016;43:504-511.

47. Sawicki LM, Grueneisen J, Buchbender C, et al. Evaluation of the outcome of lung nodules missed on ${ }^{18} \mathrm{~F}$-FDG PET/MRI compared with ${ }^{18} \mathrm{~F}-\mathrm{FDG}$ PET/CT in patients with known malignancies. J Nucl Med. 2016;57:15-20.

48. Burris NS, Johnson KM, Larson PE, et al. Detection of small pulmonary nodules with ultrashort echo time sequences in oncology patients by using a PET/MR system. Radiology. 2016;278:239-246.

49. Kirchner J, Deuschl C, Grueneisen J, et al. ${ }^{18}$ F-FDG PET/MRI in patients suffering from lymphoma: how much MRI information is really needed? Eur J Nucl Med Mol Imaging. 2017;44:1005-1013.

50. Grueneisen J, Schaarschmidt BM, Heubner M, et al. Implementation of FASTPET/MRI for whole-body staging of female patients with recurrent pelvic malignancies: a comparison to PET/CT. Eur J Radiol. 2015;84:2097-2102.

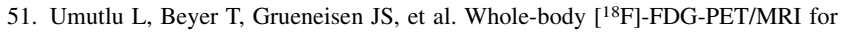
oncology: a consensus recommendation. Rofo. 2019;191:289-297.

52. Umutlu L, Beyer T, Grueneisen JS, et al. Whole-body $\left[{ }^{18} \mathrm{~F}\right]$-FDG-PET/MRI for oncology: a consensus recommendation. Nuklearmedizin. 2019;58:68-76.

53. Foster KR, Moulder JE, Budinger TF. Will an MRI examination damage your genes? Radiat Res. 2017;187:1-6.

54. Fatahi M, Reddig A, Vijayalaxmi, et al. DNA double-strand breaks and micronuclei in human blood lymphocytes after repeated whole body exposures to $7 \mathrm{~T}$ magnetic resonance imaging. Neuroimage. 2016;133:288-293.

55. Niemeyer CEA. Paediatrische Haematologie und Onkologie. New York, NY: Springer; 2018:125-132.

56. Hahn S, Hecktor J, Grabellus F, et al. Diagnostic accuracy of dual-time-point ${ }^{18} \mathrm{~F}-\mathrm{FDG}$ PET/CT for the detection of axillary lymph node metastases in breast cancer patients. Acta Radiol. 2012;53:518-523. 